\title{
Zygapophyseal Joint Orientation and Facet Tropism and Their Association with Lumbar Disc Prolapse
}

\author{
Simanchal Prosad Mohanty ${ }^{1}$, Madhava Pai Kanhangad ${ }^{1}$, Siddarth Kamath ${ }^{1}$, Asha Kamath ${ }^{2}$ \\ ${ }^{1}$ Department of Orthopaedics, Kasturba Medical College, Manipal Academy of Higher Education, Manipal, India \\ ${ }^{2}$ Department of Community Medicine, Kasturba Medical College, Manipal Academy of Higher Education, Manipal, India
}

\begin{abstract}
Study Design: Cross-sectional study.
Purpose: To evaluate the association between zygapophyseal joint angle (ZJA), facet tropism (FT), and lumbar intervertebral disc prolapse (IVDP).

Overview of Literature: Several studies have shown that FT increases the risk of IVDP and have postulated that a more sagittally oriented zygapophyseal joint provides less mechanical resistance to axial torque, thereby exerting excessive rotational strain on the intervertebral disc, resulting in an annular tear. In contrast, other studies have found no definitive association between FT and IVDP. Therefore, conclusive evidence regarding the role of $\mathrm{FT}$ in the pathogenesis of disc prolapse is currently lacking.

Methods: Magnetic resonance imaging scans of 426 patients with single-level lumbar IVDP were analyzed. Right and left ZJAs of the lumbar segments were measured on axial sections. The frequency and severity of FT were determined by calculating the absolute difference between the right and left ZJAs. Patients without IVDP at L4-L5 and L5-S1 served as controls for those with IVDP at L4-L5 and $\mathrm{L} 5-\mathrm{S} 1$, respectively. Chi-square test and $t$-test were used to compare the severity and frequency of $\mathrm{FT}$ between patients with and without IVDP. The receiver operating characteristic analysis was performed to determine the critical FT values that were predictive of IVDP.

Results: Patients with IVDP exhibited a higher frequency (L4-L5: $47 \%$ vs. 15.08\%; L5-S1: $39.62 \%$ vs. $22.69 \% ; p=0.001$ ) and severity (L4-L5: $7.85^{\circ} \pm 3.5^{\circ}$ vs. $4.05^{\circ} \pm 2.62^{\circ}$; L5-S1: $7.30^{\circ} \pm 3.07^{\circ}$ vs. $4.82^{\circ} \pm 3.29^{\circ} ; p<0.001$ ) of FT than those without IVDP. Critical FT values of $5.7^{\circ}$ at $L 4-\mathrm{L} 5$ and $6^{\circ}$ at $L 5-S 1$ increased the likelihood of IVDP by a factor of 2.89 and 1.75 , respectively.

Conclusions: Our results confirm the existence of a significant association between lumbar IVDP and FT; however, a causal relationship could not be ascertained.
\end{abstract}

Keywords: Lumbar spine; Zygapophyseal joint; Facet tropism; Intervertebral disc prolapse; Magnetic resonance imaging

\section{Introduction}

Lumbar intervertebral disc prolapse (IVDP) is one of the most common causes of back pain [1] and its development is favored by abnormal mechanical loading as well as the intrinsic biological properties of the disc [2,3]. Besides providing stability to the spine, zygapophyseal joints (ZJs) are intricately involved in spine mobility. In the lumbar spine, the range of movements at each motion segment is determined by the orientation of ZJs [4]. In the upper

Received Dec 18, 2017; Revised Dec 26, 2017; Accepted Jan 9, 2018

Corresponding author: Madhava Pai Kanhangad

Division of Spine Surgery, Department of Orthopaedics, Kasturba Medical College, Manipal Academy of Higher Education, Manipal 576-104, Karnataka, India

Tel: +91-820-2922754, Fax: +91-820-2571934, E-mail: kmadhavapai@gmail.com, madhava.pai@manipal.edu 
lumbar spine, ZJs are more sagittally oriented, whereas at the lower levels, they are more coronally oriented to facilitate increased movements $[4,5]$. Biomechanical studies have shown that $Z$ J also protects the motion segment from excessive shear, flexion, and rotational forces [6]. ZJ supports $35 \%$ of the static and $33 \%$ of the dynamic compressive load on the spine [7].

Abnormal orientation, in the form of articular tropism of the ZJ, may restrict motion segment mobility, causing pain whenever movement is attempted. Facet tropism (FT) is defined as an absolute difference of more than $7^{\circ}$ between the right and left ZJ angles (ZJAs) [8]. Various authors have reported an association between FT and different spinal disorders [9-12]. FT markedly alters the movement patterns of the lumbar spine, leading to some form of segmental instability [13]. It is well accepted that an abnormal ZJA can lead to early osteoarthrosis of ZJs [10] and degenerative spondylolisthesis [11]; however, it is uncertain whether an asymmetry in ZJ by itself can produce IVDP

There is cconflicting evidence regarding the association between FT and IVDP. Several studies have shown that FT increases the risk of IVDP and have postulated that more sagittally oriented ZJ provides less mechanical resistance to axial torque, thereby exerting excessive rotational strain on the intervertebral disc, resulting in an annular tear [13-16]. In contrast, other studies have failed to find a definitive association between FT and IVDP $[8,17,18]$. Currently, there is no conclusive evidence regarding the role of FT in the pathogenesis of IVDP. A better understanding of the association of ZJ orientation and FT with IVDP may help develop specific preventive and rehabilitative strategies in patients with FT. Therefore, we aimed to investigate the association between ZJA, FT, and lumbar IVDP and to determine whether individuals with FT were at a greater risk of developing IVDP.

\section{Materials and Methods}

In this cross-sectional study, we analyzed magnetic resonance imaging (MRI) scans of 624 consecutive patients aged $<50$ years who presented between January 2012 and December 2016 with clinical features suggestive of lumbar IVDP such as low back pain and radiculopathy, with or without neurological deficits. The patients were part of a study comparing different therapeutic approaches in the management of IVDP. Preliminary investigations included standing anteroposterior and lateral flexionextension radiographs of the lumbar spine to rule out gross radiological abnormalities. Furthermore, hematological investigations were performed to rule out systemic disorders and infections. Of all, 198 patients with multilevel disc prolapse, congenital anomalies, transitional vertebrae, trauma, tumors, instability, infection, previous spine surgery, and systemic disorders (like seronegative arthropathy) were excluded, resulting in inclusion of a total of 426 patients with single-level lumbar IVDP whose MRI scans were analyzed. Study protocol approval was obtained from the institutional ethics committee (registration no., ECR/146/Inst/KA/2013). The research methodology was in compliance with the Helsinki Declaration. And informed consent was obtained from all individual participants included in the study.

\section{Evaluation of zygapophyseal joint orientation and facet tropism}

All MRIs were performed using a Philips Achieva $1.5 \mathrm{~T}$ device (Philips, Amsterdam, Netherlands) with a slice thickness of $4 \mathrm{~mm}$, and the resulting digitalized scans were analyzed using image analysis software Digimizer ver. 4.6.1 (MedCalc Software, Ostend, Belgium). The orientations of the right and left ZJs were measured from an appropriate mid-disc cut, which was parallel to the inferior endplate of the superior vertebra in which both ZJs were seen. ZJA was measured using axial T2-weighted MRI scans according to the method described by Noren et al. [15]. ZJA was defined as the angle formed by the intersection of the line connecting the anteromedial and posterolateral ends of $\mathrm{ZJ}$ and the mid-sagittal line passing through the spinous process (Fig. 1). The right and left ZJAs were measured at L1-L2, L2-L3, L3-L4, L4-L5 and L5-S1. Continuous FT was defined as the absolute difference between the right and left ZJAs. Additionally, combining the definitions of Noren et al. [15] and Vanharanta et al. [8], we categorized FT as mild $\left(5^{\circ}-7^{\circ}\right)$, moderate $\left(7^{\circ}-15^{\circ}\right)$, and severe $\left(>15^{\circ}\right)$.

\section{Evaluation of intervertebral disc prolapse}

MRI scans were analyzed using Insta-RISPACS software (Meddiff Technologies Pvt. Ltd., Bangalore, India) to determine the level, side, and type of IVDP. The level of disc prolapse was described as L1-L2, L2-L3, L3-L4, L4- 
L5, and L5-S1, and the side of the prolapse was noted as left, right, and central. The prolapse type was classified as central, paracordal, and pararhizal, and its severity was graded as bulge, protrusion, extrusion, and sequestration [19].

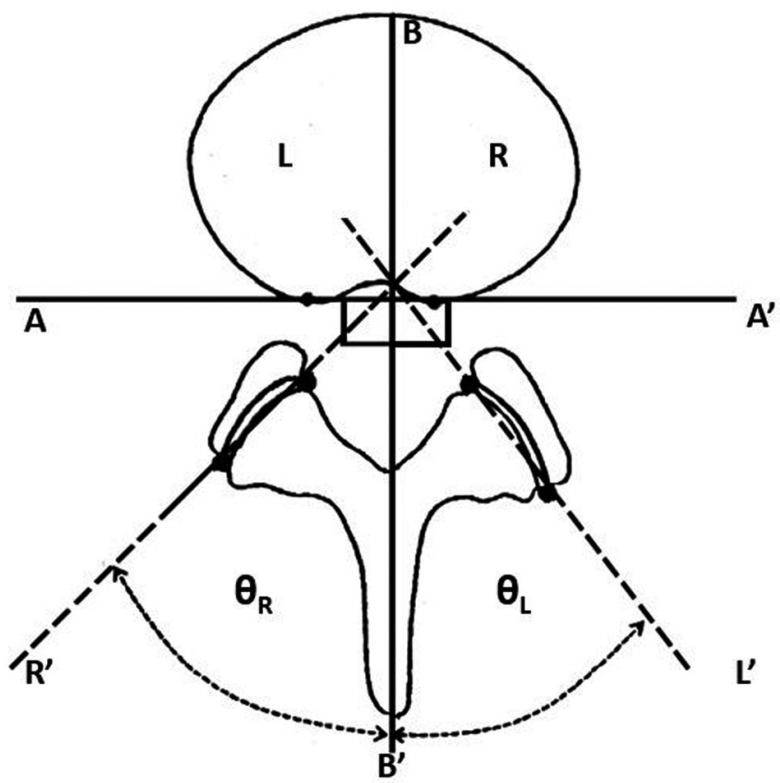

Fig. 1. Schematic diagram showing ZJA measurement. A reference line drawn along the posterior border of the vertebral body in the coronal plane $\left(A A^{\prime}\right)$. A sagittal line, drawn through the spinous process, perpendicular to the reference line $\left(\mathrm{BB}^{\prime}\right)$. Intersecting lines connecting the anteromedial and posterolateral ends of each zygapophyseal joint on the right and left side (RR' and $\left.\mathrm{LL}^{\prime}\right)$. ZJAs on the right and left side ( $\theta R$ and $\theta \mathrm{L})$. ZJA, zygapophyseal joint angle.

\section{Reliability of magnetic resonance imaging readings}

All MRIs were analyzed by two independent observers (MPK and SK) in a blinded fashion. In case of a difference in ZJA readings, the final ZJA was the mean of the two readings. Inter- and intra-observer variabilities were evaluated using the intra-class correlation coefficient., The intra- and inter-observer variabilities for ZJA and IVDP evaluation ranged from 0.93 to 0.97 and from 0.91 to 0.96 , respectively.

\section{Statistical analysis}

Statistical analyses were performed using the SPSS ver. 15.0 (SPSS Inc., Chicago, IL, USA). Continuous variables such as age, the mean ZJA, and FT were expressed as mean \pm standard deviation, whereas categorical variables such as gender and side, type, and severity of IVDP were expressed as percentage. The mean ZJA, FT, and frequency of FT were compared between patients with and without disc prolapse at L4-L5 and L5-S1. An independentsample $t$-test was used to compare the means between two groups of continuous variables, whereas one-way repeated analysis of variance was used to compare more than two groups of continuous variables. Chi-square test was used to compare categorical variables. The receiver operating characteristic (ROC) curve was used to assess the area under the curve (AUC) of the mean ZJA and FT in relation to L4-L5 and L5-S1 IVDP. All $p<0.05$ were considered to

Table 1. Descriptive statistics of the studied sample $(n=426)$

\begin{tabular}{|c|c|c|c|}
\hline Spinal level & Male $(n=289)$ & Female $(n=137)$ & Comparison $^{\text {a) }} p$-value \\
\hline ZJA L1-L2 & $24.20^{\circ} \pm 6.37^{\circ}$ & $25.18^{\circ} \pm 6.64^{\circ}$ & 0.146 \\
\hline ZJA L2-L3 & $29.3^{\circ} \pm 7.3^{\circ}$ & $29.8^{\circ} \pm 7.1^{\circ}$ & 0.464 \\
\hline ZJA L3-L4 & $38.7^{\circ} \pm 7.3^{\circ}$ & $40.0^{\circ} \pm 7.4^{\circ}$ & 0.095 \\
\hline ZJA L4-L5 & $50.0^{\circ} \pm 6.8^{\circ}$ & $49.5^{\circ} \pm 6.3^{\circ}$ & 0.521 \\
\hline ZJA L5-S1 & $54.0^{\circ} \pm 6.4^{\circ}$ & $53.9^{\circ} \pm 6.6^{\circ}$ & 0.902 \\
\hline FT L1-L2 & $3.26^{\circ} \pm 2.6^{\circ}$ & $3.61^{\circ} \pm 2.72^{\circ}$ & 0.208 \\
\hline FT L2-L3 & $3.68^{\circ} \pm 2.78^{\circ}$ & $3.98^{\circ} \pm 3.23^{\circ}$ & 0.323 \\
\hline FT L3-L4 & $3.82^{\circ} \pm 2.9^{\circ}$ & $3.37^{\circ} \pm 2.5^{\circ}$ & 0.116 \\
\hline FT L4-L5 & $6.96^{\circ} \pm 3.89^{\circ}$ & $6.29^{\circ} \pm 3.22^{\circ}$ & 0.097 \\
\hline FT L5-S1 & $5.37^{\circ} \pm 3.49^{\circ}$ & $5.39^{\circ} \pm 3.26^{\circ}$ & 0.83 \\
\hline Frequency of FT (difference $>7^{\circ}$ ) in at least one level & $156(53.97)$ & 78 (56.93) & 0.67 \\
\hline
\end{tabular}

Values are presented as mean \pm standard deviation or number (\%).

ZJA, zygapophyseal joint angle; FT, facet tropism.

${ }^{a}$ Results of independent $t$-test for continuous variables and chi-square test for categorical variables. 
be significant. Post hoc power analysis was performed to ensure the validity of the results.

\section{Results}

The descriptive statistics of the participants arepresented in Table 1. Significant differences regarding the mean ZJA and FT were found among different lumbar levels. Lower spinal segments presented a more coronal ZJ. The mean age of males and females was $37.78 \pm 10.07$ and $38.47 \pm 9.24$ years, respectively. No significant differences were found between males and females regarding the mean ZJA and $\mathrm{FT}$ at the corresponding levels. The frequency of FT $>7^{\circ}$ at L4-L5 was $40.1 \%$ in males and $32.2 \%$ in females, whereas at L5-S1, it was $26.6 \%$ in males and $28.5 \%$ in females. At L4-L5, the frequencies of mild, moderate, and severe FT were $29.6 \%, 36.2 \%$, and $3.8 \%$, respectively, whereas at L5S1, they were $25.6 \%, 25.1 \%$, and $1.2 \%$, respectively.

Furthermore, IVDP occurred more frequently at L4L5 ( $\mathrm{n}=300,70.42 \%)$ and L5-S1 ( $\mathrm{n}=106,24.88 \%)$. Only 20 cases $(4.69 \%)$ of IVDP were seen at other levels. Therefore, detailed statistical analyses were performed only at L4-L5 and L5-S1. A significant difference was found regarding the age of occurrence of IVDP; compared to L4-L5, L5S1 disc prolapse occurred more commonly in younger individuals $(p=0.005)$. Univariate analysis did not show any significant difference between age groups regarding the mean ZJA, FT, and frequency of FT at L4-L5 and L5-S1. Moreover, no significant differences were found regarding the frequency of disc prolapse between males and females at L4-L5 and L5-S1 ( $p=0.54)$.
Unherniated segments were used as controls to determine the association between the mean ZJA, FT, frequency of FT, and IVDP for the corresponding herniated segments; 126 patients with unherniated L4-L5 segments

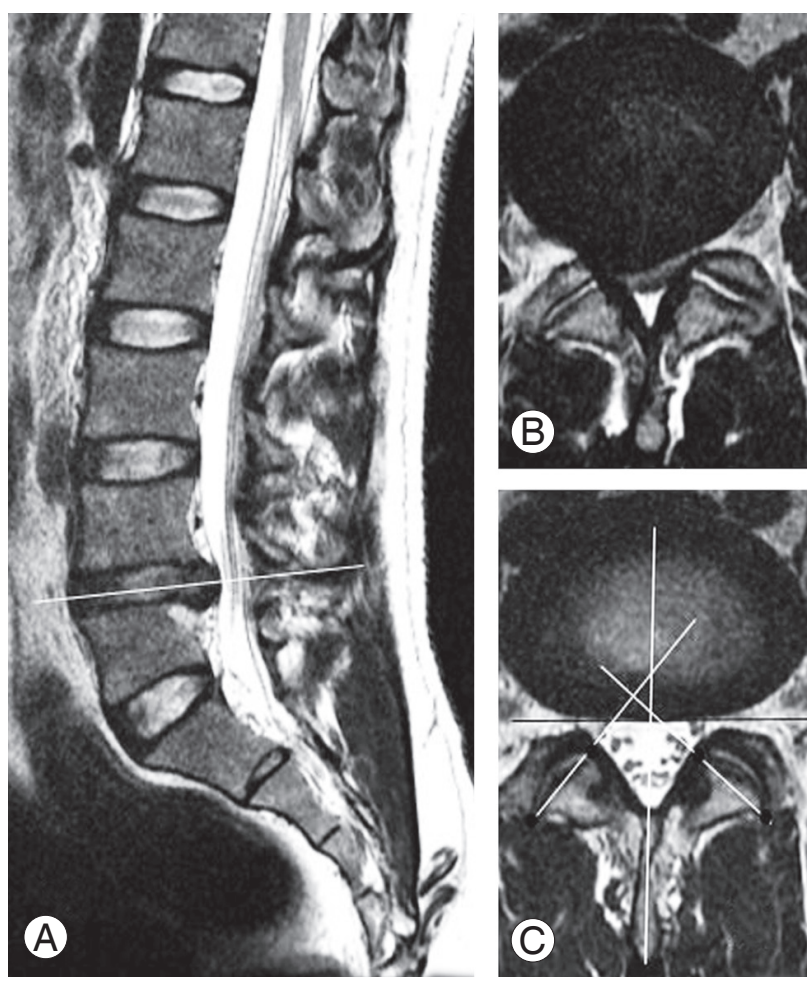

Fig. 2. Sagittal T2-weighted magnetic resonance imaging scan showing the level of the mid-disc cut at $L 4-L 5$ (A). The corresponding T2weighted axial section showing right-sided $L 4-L 5$ disc prolapse with facet tropism (B). T2-weighted axial magnetic resonance imaging scan showing an unherniated L4-L5 level (control) with the lines for the measurement of zygapophyseal joint angles (C).

Table 2. Association between ZJA, FT and frequency of FT and intervertebral disc prolapse at L4-L5 and L5-S1

\begin{tabular}{lccc} 
Variable & Disc prolapse present & Disc prolapse absent & Comparison ${ }^{\text {a) }} p$-value \\
\hline No. of L4-L5 & 300 & 126 & \\
\hline No. of L5-S1 & 106 & 320 & \\
\hline Spinal level & & & \\
\hline ZJA L4-L5 & $49.97^{\circ} \pm 6.6^{\circ}$ & $49.70^{\circ} \pm 6.8^{\circ}$ & 0.706 \\
\hline ZJA L5-S1 & $54.08^{\circ} \pm 6.7^{\circ}$ & $53.99^{\circ} \pm 6.45^{\circ}$ & 0.901 \\
\hline FT L4-L5 & $7.85^{\circ} \pm 3.5^{\circ}$ & $4.05^{\circ} \pm 2.62^{\circ}$ & $<0.001$ \\
\hline FT L5-S1 & $7.30^{\circ} \pm 3.07^{\circ}$ & $4.82^{\circ} \pm 3.29^{\circ}$ & $<0.001$ \\
\hline Frequency of FT L4-L5 & $141(47)$ & $19(15.08)$ & $<0.001$ \\
\hline Frequency of FT L5-S1 & $42(39.62)$ & $74(22.69)$ & 0.001 \\
\hline
\end{tabular}

Values are presented as mean \pm standard deviation or number (\%).

ZJA, zygapophyseal joint angle; FT, facet tropism.

${ }^{a}$ Results of independent $t$-test for continuous variables and chi-square test for categorical variables. 
acted as controls for 300 patients with IVDP at the same level (Fig. 2), whereas 320 patients with unherniated L5S1 segments acted as controls for 106 patients with IVDP at the same level. At L4-L5 and L5-S1, no significant difference was found regarding the mean ZJA between patients with and without IVDP; however, a significant difference regarding FT was found at both these levels $(p<0.001)$. Chi-square test revealed a significant difference between patients with and without IVDP regarding the frequency of FT both at L4-L5 $(p<0.001)$ and L5-S1 $(p=0.001)$ (Table 2). Post hoc analysis revealed a power range between $94 \%$ and $99 \%$.

Subgroup analysis revealed no association between the side of IVDP and the side of the more sagittally oriented ZJ at L4-L5 and L5-S1 (Fig. 3). No significant differences were found regarding the mean ZJA, FT, and frequency of FT between the right- and left-sided IVDPs at L4-L5 and L5-S1. Similar findings were observed in patients with
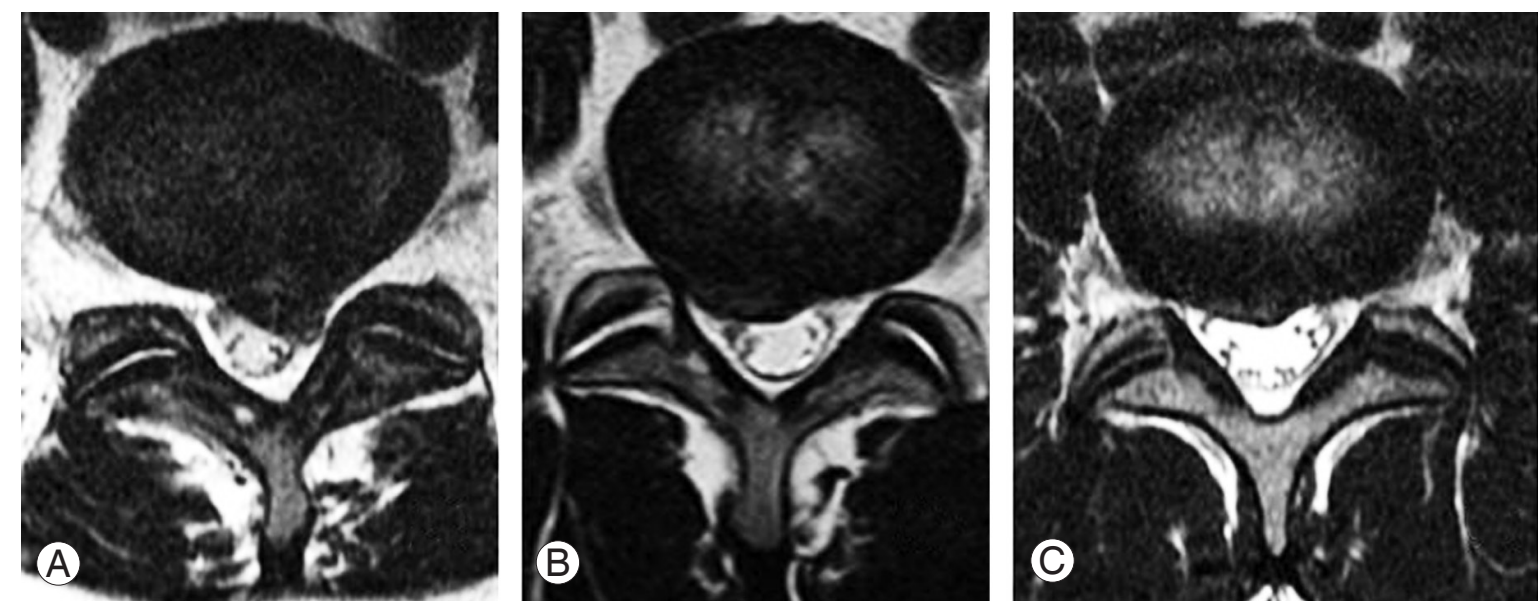

Fig. 3. T2-weighted axial magnetic resonance imaging scan showing left- and right-sided L5-S1 disc prolapse with FT (A, B). Central L5-S1 intervertebral disc prolapse was not associated with FT (C). FT, facet tropism.

Table 3. Sub-group analyses for determining the association between side of IVDP and the side of the more sagittal zygapophyseal joint

\begin{tabular}{|c|c|c|c|c|}
\hline Disc prolapse & Right sided IVDP & Left sided IVDP & Central IVDP & Comparison $^{\text {a) }} p$-value \\
\hline L4-L5 (n=300) & L4-L5 (n=78) & L4-L5 (n=90) & L4-L5 (n=132) & \\
\hline L5-S1 (n=106) & L5-S1 (n=30) & L5-S1 (n=28) & L5-S1 (n=48) & \\
\hline L4-L5 right ZJA & $49.71^{\circ} \pm 8.14^{\circ}$ & $50.94^{\circ} \pm 7.71^{\circ}$ & $49.37^{\circ} \pm 6.87^{\circ}$ & 0.29 \\
\hline L4-L5 left ZJA & $49.8^{\circ} \pm 8.75^{\circ}$ & $50.45^{\circ} \pm 6.56^{\circ}$ & $49.81^{\circ} \pm 9.1^{\circ}$ & 0.83 \\
\hline L4-L5 mean ZJA & $49.76^{\circ} \pm 7.35^{\circ}$ & $50.69^{\circ} \pm 5.83^{\circ}$ & $49.6^{\circ} \pm 6.69^{\circ}$ & 0.46 \\
\hline L4-L5 right facet more sagittal & $39(50)$ & $45(50)$ & $67(50.8)$ & 1 \\
\hline L4-L5 left facet more sagittal & $39(50)$ & $45(50)$ & $65(49.2)$ & 1 \\
\hline L4-L5 mean FT & $7.61^{\circ} \pm 3.34^{\circ}$ & $7.43^{\circ} \pm 3.66^{\circ}$ & $8.27^{\circ} \pm 3.47^{\circ}$ & 0.16 \\
\hline L4-L5 frequency of FT & $30(38.5)$ & $38(42.2)$ & $73(55.3)$ & 0.034 \\
\hline L5-S1 right ZJA & $52.73^{\circ} \pm 6.59^{\circ}$ & $53.74^{\circ} \pm 7.26^{\circ}$ & $53.23^{\circ} \pm 7.28^{\circ}$ & 0.86 \\
\hline L5-S1 left ZJA & $54.61^{\circ} \pm 8.82^{\circ}$ & $54.56^{\circ} \pm 9.11^{\circ}$ & $55.36^{\circ} \pm 7.87^{\circ}$ & 0.89 \\
\hline L5-S1 mean ZJA & $53.67^{\circ} \pm 6.28^{\circ}$ & $54.16^{\circ} \pm 7.11^{\circ}$ & $54.3^{\circ} \pm 6.85^{\circ}$ & 0.92 \\
\hline L5-S1 right facet more sagittal & $18(60)$ & $15(53.6)$ & $31(64.6)$ & 0.638 \\
\hline L5-S1 left facet more sagittal & $12(40)$ & $13(46.4)$ & $17(35.4)$ & 0.638 \\
\hline L5-S1 mean FT & $8.63^{\circ} \pm 3.33^{\circ}$ & $7.87^{\circ} \pm 2.39^{\circ}$ & $6.16^{\circ} \pm 2.87^{\circ}$ & 0.001 \\
\hline L5-S1 frequency of FT & $16(53.3)$ & $15(53.6)$ & $11(22.9)$ & 0.006 \\
\hline
\end{tabular}

Values are presented as mean \pm standard deviation or number (\%).

IVDP, intervertebral disc prolapse; ZJA, zygapophyseal joint angle; FT, facet tropism.

${ }^{a}$ Results of one way repeated analysis of variance for continuous variables and chi-square test for categorical variables. 
central IVDP at L4-L5. However, in patients with central IVDP at L5-S1, a negative association with FT was observed ( $p=0.001$ ) (Table 3 ). This suggests that at L5-S1, in contrast to right and left IVDPs, central IVDP is unlikely to occur in the presence of FT. There was a trend toward higher FT values in more severe grades of IVDP at both L4-L5 and L5-S1.

The ROC analysis revealed an AUC of 0.51 for the mean ZJA at L4-L5 and L5-S1 and an AUC of 0.8 for FT at L4L5 and 0.71 at L5-S1 in relation to IVDP at these levels (Fig. 4). The analysis showed that continuous FT was a better parameter than the mean ZJA to discriminate between patients with and without disc prolapse. In all types of IVDP, the critical FT value at L4-L5 was $5.7^{\circ}$, with a sensitivity of $78 \%$, specificity of $73 \%$, positive predictive value of $87.3 \%$, and negative predictive value of $58.2 \%$. At L5-S1, the critical FT value was $6^{\circ}$, with a sensitivity of $70.8 \%$, specificity of $59.8 \%$, positive predictive value of $36.8 \%$, and negative predictive value of $86 \%$ for rightand left-sided IVDPs. However, the critical value of $6^{\circ}$ had poor sensitivity, specificity, and positive predictive value for central IVDP at L5-S1 due to its negative association with FT. Critical FT values of $5.7^{\circ}$ or more at L4-L5 and $6^{\circ}$ or more at L5-S1 increased the likelihood of IVDP by a factor of 2.89 and 1.75 , respectively.

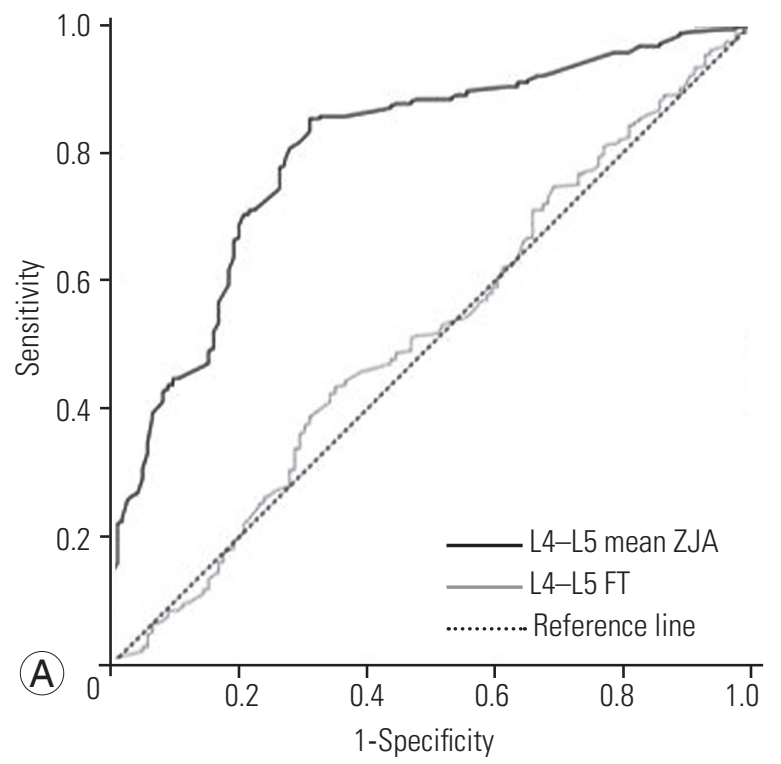

\section{Discussion}

A symmetrical alignment of the right and left ZJs is essential for their synchronous movements, which play an important role in the normal biomechanics of the spine. ZJ asymmetry, in the form of FT, results in segmental instability, leading to abnormal load on the spinal motion segment $[6,13]$. While greater sagittal ZJ orientation is associated with degenerative spondylolisthesis [11], ZJA is not considered to play a major role in the pathogenesis of IVDP. In line with the findings of previous studies, our results show a gradual increase in the mean ZJA from L1-L2 through L5-S1 [5,20].All adjacent lumbar levels differed significantly regarding the mean ZJA, making the use of adjacent unherniated spinal segments as controls for segments with IVDP unreasonable. In this study, no significant differences were found regarding the mean ZJA between patients with and without disc prolapse at L4-L5 and L5-S1, which is consistent with the findings of previous studies [13-15,18].

Since Farfan and Sullivan [14] hypothesized that FT was associated with the development of IVDP, several studies have yielded conflicting results on this topic $[8,12,13,15,17,18]$. Cyron and Hutton [13] postulated that FT could lead to segmental instability, resulting in increased rotational strain on the annulus fibrosus. Noren et al. [15] stated that FT is a risk factor in the develop-

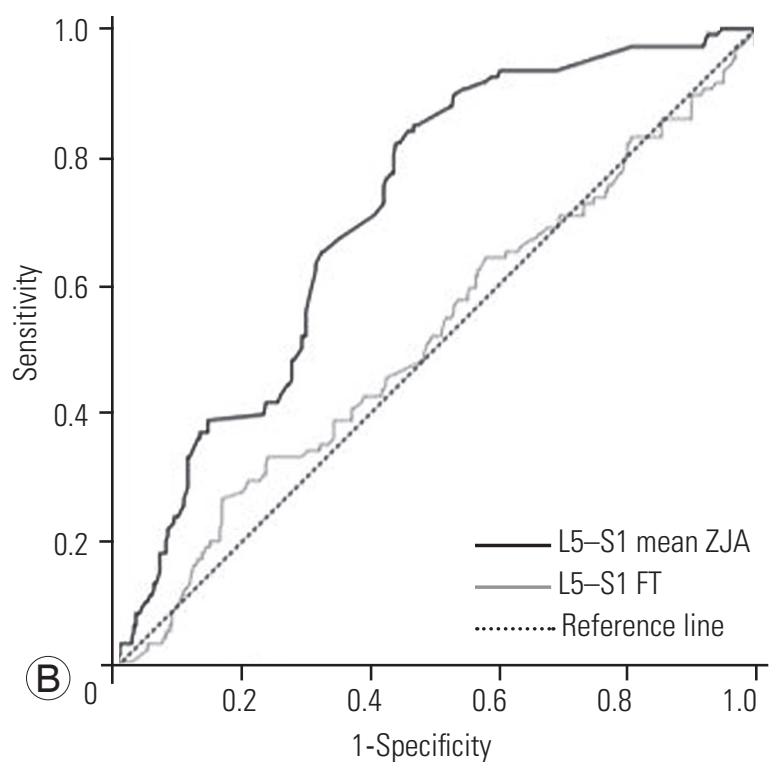

Fig. 4. The receiver operating characteristic curves for prediction of disc prolapse at $L 4-L 5(\mathbf{A})$ and $L 5-S 1$ (B). The area under the curve was greater for facet tropism than that for mean zygapophyseal joint angle at both L4-L5 and L5-S1. ZJA, zygapophyseal joint angle; FT, facet tropism. 
ment of IVDP. Although FT was observed at all lumbar levels in our study, the highest frequency of FT was noted at L4-L5 followed by L5-S1. Furthermore, we found that the highest frequency of IVDP occurred at L4-L5 followed by L5-S1. Patients with disc prolapse exhibited greater values of continuous FT and higher frequencies of FT than those without disc prolapse, which is in line with the findings of other studies [12-15]. Some authors have found a correlation between unilateral IVDP and FT; they hypothesized that uneven loading of the posterior column of the spine, due to facet asymmetry, places an additional rotational torque on the motion segment, resulting in the mechanical failure of the annulus fibrosus on the side of the more sagittally oriented ZJ $[14,16]$. In contrast, other authors found no association between the side of the more sagittally oriented ZJ and that of unilateral IVDP $[8,17,18]$. Biomechanical studies conducted by Ahmed et al. [6] showed that the more sagittally oriented ZJ neither allowed a greater axial rotation nor increased the risk of torsional injury to the annulus. In our study, subgroup analysis did not reveal any association between the side of the more sagittally oriented ZJ and that of L4-L5 and L5S1 disc prolapse. These findings can be explained in the light of other biological and mechanical factors such as the position of the spine at the time of injury, which may also determine the side of lateral herniation [2]. In the presence of an intact ZJ, axial rotation must be coupled with other movements, such as flexion, to result in posterolateral annular tears [6,16,21-23]. In the current study, central IVDP at L4-L5 was significantly associated with FT, whereas a negative association was seen at L5-S1. The L4-L5 and L5-S1 motion segments differ in terms of biomechanical characteristics. The L4-L5 intervertebral disc lies between two mobile segments, whereas the L5-S1 disc is located between a mobile and a fixed segment. An increase in inclination at $\mathrm{S} 1$ and absence of the beneficial role of extensor muscles results in an increased shear force on the L5-S1 disc $[24,25]$. Thus, the strong association observed between FT and central IVDP at L4-L5 but not at L5-S1 can be explained by a difference in the loading pattern.

The low cut-off values obtained in the ROC analysis show that an asymmetry as small as $6^{\circ}$ increases the risk of IVDP. Therefore, the presence of FT must be reported in imaging studies of the lumbar spine. Furthermore, patients with FT need to be taught specific preventive strategies to avoid injury to the intervertebral disc, whereas those undergoing treatment for disc prolapse, associated with FT, must be warned regarding the risk of recurrence and need to be educated regarding specific rehabilitative measures.

To the best of our knowledge, this is one of the largest studies investigating the association between the mean ZJA, FT, frequency of FT, and lumbar IVDP. Previous researchers have used unherniated segments adjacent to the herniated levels as controls $[12,15,18,25]$. However our study results showed a significant difference in the mean ZJA at different levels of the lumbar spine, similar to those of previous studies $[5,13,26]$. Therefore, we compared the mean ZJA and FT in patients with IVDP at L4-L5 and L5-S1 with corresponding unherniated levels, without using adjacent lumbar levels as controls. The main limitation of this study is its retrospective nature.

\section{Conclusions}

Our study demonstrates a significant association between FT and IVDP. However, no association was observed between the side of the more sagittally oriented ZJ and that of unilateral IVDP. Central IVDP showed a positive association with FT at L4-L5, whereas a negative association was present between the two at L5-S1.

\section{Conflict of Interest}

No potential conflict of interest relevant to this article was reported.

\section{References}

1. Katz JN. Lumbar disc disorders and low-back pain: socioeconomic factors and consequences. J Bone Joint Surg Am 2006;88 Suppl 2:21-4.

2. Adams MA, Roughley PJ. What is intervertebral disc degeneration, and what causes it? Spine (Phila $\mathrm{Pa}$ 1976) 2006;31:2151-61.

3. Nachemson A. Lumbar intradiscal pressure: experimental studies on post-mortem material. Acta Orthop Scand Suppl 1960;43:1-104.

4. Taylor JR, Twomey LT. Structure and function of lumbar zygapophyseal (facet) joints: a review. J Orthop Med 1992;14:71-8.

5. White AA, Panjabi MM. Clinical biomechanics of the spine. 2nd ed. Philadelphia (PA): Lippincott Williams 
\& Wilkins; 1990.

6. Ahmed AM, Duncan NA, Burke DL. The effect of facet geometry on the axial torque-rotation response of lumbar motion segments. Spine (Phila Pa 1976) 1990;15:391-401.

7. Lorenz M, Patwardhan A, Vanderby R Jr. Loadbearing characteristics of lumbar facets in normal and surgically altered spinal segments. Spine (Phila Pa 1976) 1983;8:122-30.

8. Vanharanta H, Floyd T, Ohnmeiss DD, Hochschuler $\mathrm{SH}$, Guyer RD. The relationship of facet tropism to degenerative disc disease. Spine (Phila $\mathrm{Pa}$ 1976) 1993;18:1000-5.

9. Ghormley RK. Low back pain: with special reference to the articular facets, with presentation of an operative procedure. JAMA 1933;101:1773-7.

10. Kalichman L, Suri P, Guermazi A, Li L, Hunter DJ. Facet orientation and tropism: associations with facet joint osteoarthritis and degeneratives. Spine (Phila $\mathrm{Pa}$ 1976) 2009;34:E579-85.

11. Samartzis D, Cheung JP, Rajasekaran S, et al. Critical values of facet joint angulation and tropism in the development of lumbar degenerative spondylolisthesis: an international, large-scale multicenter study by the AOSpine Asia Pacific Research Collaboration Consortium. Global Spine J 2016;6:414-21.

12. Karacan I, Aydin T, Sahin Z, et al. Facet angles in lumbar disc herniation: their relation to anthropometric features. Spine (Phila Pa 1976) 2004;29:11326.

13. Cyron BM, Hutton WC. Articular tropism and stability of the lumbar spine. Spine (Phila Pa 1976) 1980;5:168-72.

14. Farfan HF, Sullivan JD. The relation of facet orientation to intervertebral disc failure. Can J Surg 1967;10:179-85.

15. Noren R, Trafimow J, Andersson GB, Huckman MS. The role of facet joint tropism and facet angle in disc degeneration. Spine (Phila Pa 1976) 1991;16:530-2.
16. Kim HJ, Chun HJ, Lee HM, et al. The biomechanical influence of the facet joint orientation and the facet tropism in the lumbar spine. Spine J 2013;13:1301-8.

17. Van Schaik JP, Verbiest H, van Schaik FD. The orientation of laminae and facet joints in the lower lumbar spine. Spine (Phila Pa 1976) 1985;10:59-63.

18. Cassidy JD, Loback D, Yong-Hing K, Tchang S. Lumbar facet joint asymmetry: intervertebral disc herniation. Spine (Phila Pa 1976) 1992;17:570-4.

19. Wong DA. Macnab's backache. 4th ed. Philadelphia (PA): Lippincott Williams \& Wilkins; 2007.

20. Weinberg DS, Xie KK, Liu RW, Gebhart JJ, Gordon ZL. Increased pelvic incidence is associated with a more coronal facet orientation in the lower lumbar spine: a cadaveric study of 599 lumbar spines. Spine (Phila Pa 1976) 2016;41:E1138-45.

21. Shirazi-Adl SA, Shrivastava SC, Ahmed AM. Stress analysis of the lumbar disc-body unit in compression: a three-dimensional nonlinear finite element study. Spine (Phila Pa 1976) 1984;9:120-34.

22. Adams MA, Hutton WC. The relevance of torsion to the mechanical derangement of the lumbar spine. Spine (Phila Pa 1976) 1981;6:241-8.

23. Schmidt H, Kettler A, Heuer F, Simon U, Claes L, Wilke HJ. Intradiscal pressure, shear strain, and fiber strain in the intervertebral disc under combined loading. Spine (Phila Pa 1976) 2007;32:748-55.

24. Arjmand N, Shirazi-Adl A. Biomechanics of changes in lumbar posture in static lifting. Spine (Phila $\mathrm{Pa}$ 1976) 2005;30:2637-48.

25. Chadha M, Sharma G, Arora SS, Kochar V. Association of facet tropism with lumbar disc herniation. Eur Spine J 2013;22:1045-52.

26. Mohanty SP, Pai Kanhangad M, Kamath S, Kamath A. Morphometric study of the orientation of lumbar zygapophyseal joints in a South Indian population. J Orthop Surg (Hong Kong) 2017;25:2309499017739483. 